\title{
Short-and long-term effects of ischemic postconditioning in STEMI patients: a meta-analysis
}

Jing Gao ${ }^{1,2,3 \dagger}$, Junyi Luo ${ }^{1,2 \dagger}$, Fen Liu ${ }^{2}$, Yingying Zheng ${ }^{1,2}$, Bangdang Chen ${ }^{2}$, Qingjie Chen ${ }^{1,2}$ and Yining Yang ${ }^{1,2^{*}}$

\begin{abstract}
Background: Compelling evidence from large randomized trials demonstrates the salutary effects of ischemic postconditioning on cardioprotection against ischemic/reperfusion injury. However, some studies appear negative findings.

This study was designed to assess the short-and long-term effects of postconditioning (Poc) in studies including evolving ST-elevation myocardial infarction (STEMI).

Methods: Relevant studies were identified through an electronic literature search from the PubMed, Library of Congress, Embase, Cochrane Central Register of Controlled Trials, and ISI Web of Science. Studies published up to December 2014 were eligible for inclusion. Patients older than 18 years presenting within $12 \mathrm{~h}$ of the first STEMI and eligible for angioplasty were considered for the study.

Results: The 25 trials allocated 1136 patients to perform locational postconditioning cycles at the onset of reperfusion and 1153 patients to usual percutaneous coronary intervention (PCI). Ischemic postconditioning demonstrated a decrease in serum cardiac enzymes creatine kinase (CK) and CK-MB $(P<0.00001$ and $P=0.25$, respectively) in the subgroup analysis based on direct stenting. Reduction in infarct size by imaging was showed during7 days after myocardial infarction $(P=0.01)$, but not in the longterm $(P=0.08)$. The wall motion score index was improved in both the short term within 7 days $(P=0.009)$ and the long term over 6 months after receiving Poc $(P=0.02)$. All included studies were limited by the high risk of performance and publication bias.

Conclusions: Ischemic postconditioning by brief interruptions of coronary blood flow at the onset of reperfusion after $\mathrm{PCl}$ appears to be superior to $\mathrm{PCl}$ alone in reducing myocardial injury and improving left ventricular function, especially in patients who have received direct stenting in $\mathrm{PCl}$.
\end{abstract}

Keywords: Ischemic postconditioning, STEMI, Ischemic/reperfusion injury, Cardioprotection, Infarct size

\section{Background}

Ischemic/reperfusion injury appearing after primary percutaneous coronary intervention (PCI) abrogates myocardial salvage and may increase infarct size [1]. It was proved that ischemic preconditioning, which involved a series of brief ischemia/reperfusion cycles and performed before ischemia, was explicitly a cardioprotective

\footnotetext{
* Correspondence: yangyn5126@163.com

${ }^{\dagger}$ Equal contributors

'Department of Cardiology, First Affiliated Hospital of Xinjiang Medical University, Urumqi 830054, P.R., China

${ }^{2}$ Xinjiang Key Laboratory of Cardiovascular Disease Research, Urumqi 830054, P.R., China

Full list of author information is available at the end of the article
}

strategy [2-4]. Unlike preconditioning, ischemic postconditioning, which involves brief episodes of ischemia/ reperfusion during early reperfusion, has been demonstrated to be effective in many studies [5-9]. Ischemic postconditioning has a promising potential to be applied in the clinic. However, some studies suggest that ischemic postconditioning during primary PCI does not reduce infarct size or improve myocardial function recovery $[10,11]$. The purpose of this paper was to further summarize the evidence supporting cardioprotection of ischemic postconditioning in patients with acute STEMI by conducting a meta-analysis of the published literature.

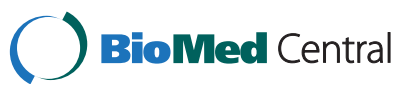

(c) 2015 Gao et al. Open Access This article is distributed under the terms of the Creative Commons Attribution 4.0 International License (http://creativecommons.org/licenses/by/4.0/), which permits unrestricted use, distribution, and reproduction in any medium, provided you give appropriate credit to the original author(s) and the source, provide a link to the Creative Commons license, and indicate if changes were made. The Creative Commons Public Domain Dedication waiver (http://creativecommons.org/publicdomain/zero/1.0/) applies to the data made available in this article, unless otherwise stated. 


\section{Methods}

\section{Search strategy}

To avoid insufficient number of studies, an electronic literature search was simultaneously conducted across the PubMed, Library of Congress, Embase, Cochrane Central Register of Controlled Trials, and ISI Web of Science. Two independent evaluators reviewed all English language articles published up to 2014. The following key words were used as search terms: ischemic postconditioning, reperfusion, ischemic reperfusion injury, primary percutaneous intervention, controlled trials, and randomized controlled trials (RCTs). All prospective, randomized, single-center, or multicenter clinical trials were included.

\section{Eligibility criteria}

Criteria for inclusion were as follows:(1) subjects with explicit STEMI, (2) two reperfusion strategies, PCI with postconditioning (Poc) or conventional (Con) PCI, were compared, (3) a similar baseline between Poc and the control group, with a good match of age, gender, ischemic time, and risk factors, (4) one or more myocardial injury-related indicators, which involve peak creatine kinase (CK), peak CK-MB, ST-segment resolution, infarct size (IS), left ventricular ejection fraction (LVEF), and wall motion score index (WMSI). Continuous variables were reported as mean \pm standard deviation (SD). Only the latest study was included in the meta-analysis for identical or largely similar articles.

\section{Study selection and quality assessment}

Two investigators independently assessed the eligibility of identified studies. The studies that were evaluated were RCTs that focused on the role of Poc in STEMI. Published abstracts or without data were excluded. Disagreement resolved by discussion or by referral to a third assess or if necessary. Complete consensus among the authors on the final results was achieved. Studies included in the meta-analysis had to fulfill the aforementioned eligibility criteria. The criteria for study quality outlined by the Cochrane Reviewer's Handbook 4.2were adopted for quality assessment of included RCTs. These criteria were as follows: (1) correct random methods, (2) randomization, (3) blindness assessment, (4) completeness of the follow-up and using ITT (intention-to-treat) analysis to deal with the dropouts.

\section{Statistical analysis}

Continuous data were reported either as mean (SD) or median (interquartile range). For continuous data, mean difference was calculated where same scale was used to measure relevant outcomes (peak CK, peak CK-MB, LV EF, and WMSI). A random effects model was used to pool data, and the corresponding forest plots were constructed. The Cochran's Q test was used to assess the heterogeneity among studies and was complemented by the $I^{2}$ statistic [12]. All analyses were conducted using the statistical software Review Manager (RevMan) version 5.3. The authors are solely responsible for the design and conduct of this study and its final contents.

\section{Results}

\section{Identification of studies}

A total of 25 eligible publications were screened by the investigators. The studies that were excluded were as follows: (1) 949 manuscripts based on titles and abstracts and (2) 27 articles that either lacked original data $[5,13,14]$, or were with inaccurate data [15], or involved remote postconditioning [16-18] and pharmacological postconditioning [19-24], or involved patients who were not suffering from STEMI and treated with PCI [6, 25-37]. The studies were excluded based on the full-text review. Twenty-five studies [7-11, 38-57] were included in this metaanalysis (Fig. 1).

\section{Study characteristics}

Among the 2289 participants included in the metaanalysis, 1136 patients were in the postconditioning group and 1153 in the conventional care group. Characteristics of studies and patients are summarized in Tables 1 and 2 .

The Poc protocol (cycles $\times$ ischemia/reperfusion in seconds) varied between studies, being $2 \times 90^{\prime \prime} / 180$ " in 1 study, $10 \times 30^{\prime \prime} / 30^{\prime \prime}$ in 1 study, $3 \times 30 " / 30^{\prime \prime}$ to $4 \times$ $30 " / 30^{\prime \prime}$ in 9 studies, and $3 \times 60^{\prime \prime} / 60$ " to $4 \times 60^{\prime \prime} / 60$ " in 14 studies. The follow-up in the trials varied from 3 days to 3.4 years. The relevant outcomes in all studies include markers of cardiac injury and left ventricular function (Table 2).

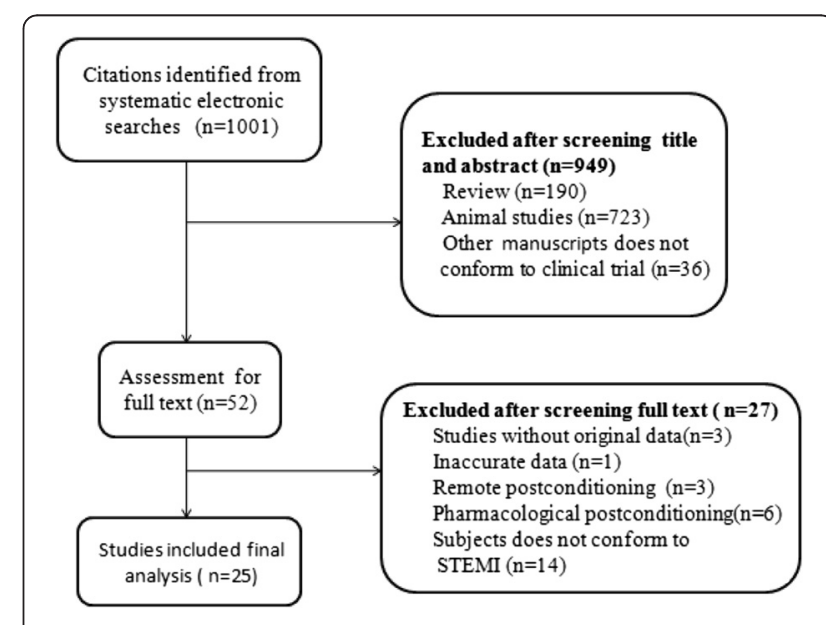

Fig. 1 Flow diagram for the selection of articles 
Table 1 Characteristics of studies included in the meta-analysis

\begin{tabular}{|c|c|c|c|c|c|c|c|c|c|}
\hline Author & Country & Year & $\begin{array}{l}\text { Number } \\
\text { (Poc/Con) }\end{array}$ & $\begin{array}{l}\text { Male (\%) } \\
\text { (Poc/Con) }\end{array}$ & $\begin{array}{l}\text { Age (y) } \\
\text { (Poc/Con) }\end{array}$ & $\begin{array}{l}\text { Dyslipidemia (\%) } \\
\text { (Poc/Con) }\end{array}$ & $\begin{array}{l}\text { Diabetes (\%) } \\
\text { (Poc/Con) }\end{array}$ & $\begin{array}{l}\text { Smokers (\%) } \\
\text { (Poc/Con) }\end{array}$ & $\begin{array}{l}\text { Hypertension (\%) } \\
\text { (Poc/Con) }\end{array}$ \\
\hline Staat [38] & France & 2005 & $16 / 14$ & $75 / 93$ & $58 / 56$ & $80 / 50$ & $20 / 13$ & $57 / 56$ & $38 / 36$ \\
\hline Ma [9] & China & 2006 & $47 / 47$ & $66 / 71$ & $64 / 64$ & NR & $38 / 45$ & NR & $62 / 55$ \\
\hline Ma [39] & China & 2007 & $32 / 29$ & $66 / 70$ & $64 / 64$ & NR & $38 / 45$ & NR & $59 / 55$ \\
\hline Yang [7] & China & 2007 & 23/18 & $87 / 61$ & $59 / 63$ & $61 / 56$ & $26 / 28$ & $61 / 50$ & $70 / 61$ \\
\hline Laskey [40] & USA & 2008 & $12 / 12$ & $58 / 58$ & $60 / 58$ & $58 / 75$ & $42 / 42$ & NR & $75 / 83$ \\
\hline Thibault [41] & France & 2008 & $17 / 21$ & $76 / 78$ & $56 / 56$ & $52 / 49$ & $10 / 12$ & $65 / 65$ & $29 / 35$ \\
\hline $\operatorname{Lin} 1[42]^{a}$ & China & 2010 & $25 / 26$ & $84 / 65$ & $59 / 63$ & $56 / 42$ & $24 / 27$ & $56 / 50$ & $52 / 54$ \\
\hline $\operatorname{Lin} 2[42]^{a}$ & China & 2010 & $24 / 26$ & $71 / 65$ & $58 / 63$ & $67 / 42$ & $21 / 27$ & $67 / 50$ & $58 / 54$ \\
\hline Lonborg $[43,44]^{b}$ & Denmark & 2010 & $59 / 59$ & $69 / 74$ & $61 / 62$ & $46 / 41$ & $7 / 7$ & $61 / 49$ & $37 / 32$ \\
\hline Sorensson [45] & Sweden & 2010 & $38 / 38$ & $82 / 89$ & $63 / 62$ & $77 / 62$ & NR & $26 / 29$ & $16 / 29$ \\
\hline Xue [46] & China & 2010 & $23 / 20$ & $95 / 94$ & $54 / 62$ & $16 / 24$ & $21 / 29$ & $63 / 71$ & $37 / 71$ \\
\hline Garcia [47] & USA & 2011 & $22 / 21$ & $86 / 76$ & $61 / 55$ & $73 / 71$ & $5 / 19$ & $23 / 43$ & 73/71 \\
\hline Liu [48] & China & 2011 & $30 / 34$ & $73 / 68$ & $59 / 59$ & NR & $30 / 32$ & $57 / 61$ & $37 / 39$ \\
\hline Freixa [10] & Spain & 2012 & $39 / 40$ & $84 / 72$ & $59 / 60$ & $44 / 35$ & $23 / 17$ & $51 / 62$ & $49 / 50$ \\
\hline Tarantini [49] & Italy & 2012 & $37 / 38$ & $85 / 85$ & $60 / 60$ & $51 / 49$ & $18 / 3$ & $67 / 77$ & $59 / 49$ \\
\hline Thuny [8] & France & 2102 & $25 / 25$ & $76 / 72$ & $57 / 57$ & $36 / 48$ & $20 / 14$ & $68 / 64$ & $40 / 48$ \\
\hline Zhao [50] & China & 2012 & $30 / 32$ & $97 / 87$ & $57 / 62$ & $31 / 17$ & $13 / 27$ & $69 / 77$ & $50 / 67$ \\
\hline Dwyer [51] & Canada & 2013 & $50 / 52$ & $88 / 89$ & $57 / 57$ & $36 / 29$ & $6 / 14$ & $44 / 44$ & $42 / 33$ \\
\hline Elzbieciak [52] & Poland & 2013 & $18 / 21$ & $67 / 86$ & $60 / 58$ & $61 / 86$ & $22 / 24$ & $67 / 52$ & $78 / 91$ \\
\hline Hahn [53] & Korea & 2013 & $350 / 350$ & 79/75 & $60 / 60$ & $40 / 46$ & $24 / 25$ & $53 / 52$ & $46 / 46$ \\
\hline Mewton [54] & France & 2013 & $25 / 25$ & $76 / 72$ & $57 / 57$ & NR & $20 / 14$ & $68 / 64$ & $48 / 40$ \\
\hline Sorensson [55] & Sweden & 2013 & $33 / 35$ & $85 / 89$ & $63 / 62$ & $N R$ & $29 / 32$ & $27 / 26$ & $15 / 31$ \\
\hline Dong [56] & China & 2014 & $32 / 30$ & $63 / 73$ & $70 / 68$ & NR & $34 / 37$ & $41 / 50$ & $72 / 63$ \\
\hline Limalanathan [11] & Norway & 2014 & $120 / 129$ & $84 / 80$ & $61 / 60$ & NR & $4 / 2$ & $49 / 54$ & $29 / 25$ \\
\hline Waltenberger [57] & Germany & 2014 & $25 / 27$ & $68 / 70$ & $60 / 60$ & $12 / 11$ & $12 / 7$ & $60 / 55$ & $48 / 33$ \\
\hline
\end{tabular}

a Lonborg et al. published 2 articles on the same trial

bin et al. compared 60-s postconditioning with $30-5$ postconditioning and no postconditioning (routine) in this study

PCI was performed by direct stenting in some studies $[7-9,11,38-42,47-49,52,56]$. However, in other studies $[10,43-46,50,51,53,55,57]$, the choice of stent was left to the discretion of the operator. Balloon angioplasty or thrombus aspiration was also allowed if a stent could not be deployed or was considered harmful.

In the eligible studies, troponin levels were measured in eight studies $[10,11,41,44,45,49,52,56]$. Eighteen $[7,9,10,38-42,45-49,51,53,54,56,57]$ studies contained data on peak or the area under the curve of CK or CK-MB. IS was measured by single-photon emission computed tomography (SPECT) or cardiovascular magnetic resonance (CMR) in eleven studies [7, 8, 10, 11, 41, $44,46,49,51,52,57]$. Global left ventricular function as determined by LVEF was measured in nine studies by echocardiography $[7,41,42,46,47,49-52]$, in eight studies by CMR $[8,11,44,45,51,54,55,57]$, and in one study by both echocardiography and CMR [10]. Regional left ventricular function was measured by WMSI in six studies [9, 39, 42, 46, 48, 50].

\section{Study quality}

The analysis of the study quality in the 25 eligible studies is presented in Table 3. Baselines between the Poc group and the control group in all the studies were comparable. The measurement data was compared by the Student $t$ test, and count data was compared by $\chi^{2}$ test between the two groups. In terms of quality, each of these studies would be graded level $\mathrm{B}$ according to the Cochrane Reviewer's Handbook 4.2 for quality assessment of included RCTs.

\section{Biomarkers of acute myocardial injury}

The commonly measured myocardial injury biochemical markers of acute myocardialinfarction (AMI) are CK 
Table $\mathbf{2}$ Characteristics of studies included in the meta-analysis

\begin{tabular}{|c|c|c|c|c|c|c|c|c|}
\hline Author & Country & Year & $\begin{array}{l}\text { Chest } \\
\text { pain }\end{array}$ & Elapsed time $(\mathrm{m})$ & Culprit lesion & Protocal & Endpoint & Follow-up \\
\hline Staat & France & 2005 & $\leq 12 \mathrm{~h}$ & NR & $\operatorname{LAD}(38 / 43) ; \operatorname{RCA}(62 / 57)$ & $60 " / 60 " \times 4$ & CKAUC;peak CK; blush grade; STR & $3 d$ \\
\hline Ma & China & 2006 & $\leq 12 \mathrm{~h}$ & $395 \pm 150 / 426 \pm 150$ & LAD (49/53); LCX (23/17); RCA (28/30) & $30 " / 30 " \times 3$ & peak CK;peak CK-MB; WMSI & $7 \mathrm{~d} ; 2 \mathrm{~m}$ \\
\hline Ma & China & 2007 & $\leq 12 \mathrm{~h}$ & $N R$ & $\operatorname{LAD}(53 / 52) ; \operatorname{LCX}(22 / 17) ; \operatorname{RCA}(25 / 31)$ & $30 " / 30 " \times 3$ & peak CK;peak CK-MB; WMSI & $7 \mathrm{~d} ; 2 \mathrm{~m}$ \\
\hline Yang & China & 2007 & $N R$ & $312 \pm 48 / 264 \pm 42$ & $\operatorname{LAD}(65 / 61) ; \operatorname{LCX}(9 / 6) ; \operatorname{RCA}(26 / 33)$ & $30 " / 30 " \times 3$ & peakCK; CKAUC;IS; LVEF & $3 \mathrm{~d} ; 1 \mathrm{w}$ \\
\hline Laskey & USA & 2008 & $\leq 6 \mathrm{~h}$ & $228 \pm 43 / 222 \pm 54$ & LAD (100/100); LCX (0); RCA (0) & $90 " / 180 " \times 2$ & peak CK; STR & NR \\
\hline Thibault & France & 2008 & $\leq 6 \mathrm{~h}$ & $283 \pm 82 / 297 \pm 104$ & NR & $60 " / 60 " \times 4$ & CKAUC; Tnl;IS; LVEF & $6 \mathrm{~m} ; 12 \mathrm{~m}$ \\
\hline Lin & China & 2010 & $\leq 12 \mathrm{~h}$ & NR & LAD (64/62); LCX (8/8); RCA (28/30) & $30 " / 30 " \times 3$ & TNFa; LV EF (7d、1y); WMSI & $7 \mathrm{~d} ; 1 \mathrm{y}$ \\
\hline Lin & China & 2010 & $\leq 12 \mathrm{~h}$ & NR & $\operatorname{LAD}(54 / 62) ; \operatorname{LCX}(8 / 77) ; \operatorname{RCA}(38 / 31)$ & $60 " / 60 " \times 3$ & TNFa;LV EF (7d、1y); WMSI & $7 \mathrm{~d} ; 1 \mathrm{y}$ \\
\hline Lonborg & Denmark & 2010 & $\leq 12 \mathrm{~h}$ & $241 \pm 148.9 / 255 \pm 196$ & LAD (44/39); LCX (8/19); RCA (47/42) & $30 " / 30 " \times 4$ & IS;IS/AAR;LVEF;peak TnT & $3 \mathrm{~m}$ \\
\hline Sorensson & Sweden & 2010 & $\leq 6 \mathrm{~h}$ & $165 \pm 63.7 / 185 \pm 87.41$ & $\operatorname{LAD}(37 / 37) ; \operatorname{LCX}(11 / 3) ; \operatorname{RCA}(53 / 61)$ & $60 " / 60 " \times 4$ & IS/AAR; TnTAUC; LVEF & $6-9 d$ \\
\hline Xue & China & 2010 & $\leq 12 \mathrm{~h}$ & $4.1 \pm 3.0 / 5.4 \pm 3.7$ & LAD (42/59); LCX (0); RCA (58/41) & $60 " / 60 " \times 4$ & CK-MB, IS, LVEF, STR & $7 d$ \\
\hline Garcia & USA & 2011 & $\leq 12 \mathrm{~h}$ & $4.5 / 4.4$ & LAD (36/24); LCX (23/10); RCA (41/67) & $30 " / 30 " \times 4$ & peakCK; CK-MB;LVEF & $3.4 y$ \\
\hline Liu & China & 2011 & $\leq 12 \mathrm{~h}$ & $312 \pm 102 / 324 \pm 108$ & LAD (53/59); LCX (10/12); RCA (37/29) & $30 " / 30 " \times 3$ & $\begin{array}{l}\text { peak CK; Peak CK-MB; WMSI;LVEF; } \\
\text { blush grade;IS }\end{array}$ & $7 d$ \\
\hline Freixa & Spain & 2012 & $\leq 12 \mathrm{~h}$ & $326 \pm 180 / 330 \pm 211$ & LAD (51/39); LCX (NR) RCA (45/47) & $60 " / 60 " \times 4$ & $\begin{array}{l}\text { peak CK; Peak CK-MB; TnT; STR; } \\
\text { IS }(7 \mathrm{~d}, 6 \mathrm{~m})\end{array}$ & $7 \mathrm{~d} ; 6 \mathrm{~m}$ \\
\hline Tarantini & Italy & 2012 & $\leq 6 \mathrm{~h}$ & $212 \pm 85 / 194 \pm 80$ & LAD (41/44); LCX (18/8); RCA (41/49) & $60 " / 60 " \times 4$ & peak Tnl; LVEF;IS; & $30 d$ \\
\hline Thuny & France & 2102 & $\leq 12 \mathrm{~h}$ & $289 \pm 31 / 215 \pm 20$ & LAD (56/56); LCX (0/8); RCA (44/36) & $60 " / 60 " \times 4$ & IS;peak CK & $3 d$ \\
\hline Zhao & China & 2012 & $\leq 12 \mathrm{~h}$ & $309 \pm 201 / 404 \pm 191$ & LAD (53/53); LCX:NR; RCA:NR & $60 " / 60 " \times 4$ & LVEF; WMSI & $1 \mathrm{w} ; 6 \mathrm{~m}$ \\
\hline Dwyer & Canada & 2013 & $\leq 6 \mathrm{~h}$ & $150 \pm 70 / 170 \pm 84$ & LAD (50/46); LCX:(12/14); RCA:(38/40) & $30 " / 30 " \times 4$ & IS; AAR; PeakCK; LVEF & $3 d$ \\
\hline Elzbieciak & Poland & 2013 & $\leq 12 \mathrm{~h}$ & $225.6 \pm 139.4 / 317.6 \pm 195.8$ & LAD (100/100); LCX (0) RCA (0) & $60 " / 60 " \times 4$ & $\begin{array}{l}\text { IS; AAR; PeakCK-MB; peak TnT; } \\
\text { LVEF;IS/AAR }\end{array}$ & $3 \mathrm{~d} ; 6 \mathrm{~m}$ \\
\hline Hahn & Korea & 2013 & $\leq 12 \mathrm{~h}$ & $196 \pm 51 / 195 \pm 171$ & LAD (47/45); LCX:(11/11); RCA:(42/44) & $60 " / 60 " \times 4$ & peakCK-MB; STR; blush grade & $30 d$ \\
\hline Mewton & France & 2013 & $\leq 12 \mathrm{~h}$ & $289 \pm 31 / 215 \pm 20$ & LAD (56/56); LCX (0/8); RCA (44/36) & $60 " / 60 " \times 4$ & LVEF;IS;IS/AAR & $4 d$ \\
\hline Sorensson & Sweden & 2013 & $\leq 6 \mathrm{~h}$ & $165 \pm 51 / 180 \pm 84$ & LAD (33/37); LCX (9/3); RCA (57/60) & $60 " / 60 " \times 4$ & IS; LVEF & $12 \mathrm{~m}$ \\
\hline Dong & China & 2014 & $\leq 12 \mathrm{~h}$ & $300 \pm 90 / 294 \pm 66$ & LAD (56/43); LCX (6/10); RCA (38/47) & $30 " / 30 " \times 3$ & blush grade; STR; CK- MB; TnT; LVEF & $7 d ; 30 d$ \\
\hline Limalanathan & Norway & 2014 & $\leq 6 \mathrm{~h}$ & NR & LAD (46/51); LCX (13/9); RCA (41/41) & $60 " / 60 " \times 4$ & IS (CECMR); STR; TnT; LVEF & $2 \mathrm{~d} ; 4 \mathrm{~m}$ \\
\hline Waltenberger & Germany & 2014 & $\leq 6 \mathrm{~h}$ & NR & LAD (28/26); LCX (NR); RCA (64/74) & $30 " / 30 " \times 10$ & CKAUC;IS; LVEF & $4 d ; 4 \mathrm{~m} ; 12 \mathrm{~m}$ \\
\hline
\end{tabular}

NR,not related; LAD,left anterior descending branch; RCA,right coronary arterry; LCX,left circumflex artery; CK,creatine kinase; CKAUC,CK area under the curve; CK-MB,creatine kinase isoenzyme; STR,ST resolution; WMSI, wall motion score index; IS, infarct size; LVEF, left ventricular ejection fraction; Tnl,troponin I; TNFa,tumor necrosis factor; AAR,area at risk; TnT, troponin T 
Table 3 Quality assessments of studies included in the meta-analysis

\begin{tabular}{|c|c|c|c|c|c|}
\hline Study & Year & Randomization & Blinded assessment & Dropout rate (\%) & Similar baseline \\
\hline Staat & 2005 & yes & no & unclear & yes \\
\hline Ma & 2006 & yes & no & unclear & yes \\
\hline $\mathrm{Ma}$ & 2007 & yes & no & unclear & yes \\
\hline Yang & 2007 & yes & unclear & unclear & yes \\
\hline Laskey & 2008 & yes & unclear & 0 & yes \\
\hline Thibault & 2008 & yes & single-blind & 0 & yes \\
\hline $\operatorname{Lin} 1$ & 2010 & yes & no & unclear & yes \\
\hline $\operatorname{Lin} 2$ & 2010 & yes & no & unclear & yes \\
\hline Lonborg & 2010 & yes & unclear & 0.26 & yes \\
\hline Sorensson & 2010 & yes & no & 0.15 & yes \\
\hline Xue & 2010 & yes & unclear & 0 & yes \\
\hline Garcia & 2011 & yes & unclear & 0 & yes \\
\hline Liu & 2011 & yes & unclear & 0 & yes \\
\hline Freixa & 2012 & yes & no & 0 & yes \\
\hline Tarantini & 2012 & yes & no & 0.04 & yes \\
\hline Thuny & 2102 & yes & no & 0.19 & yes \\
\hline Zhao & 2012 & yes & no & 0.09 & yes \\
\hline Dwyer & 2013 & yes & unclear & 0.23 & yes \\
\hline Elzbieciak & 2013 & yes & no & 0 & yes \\
\hline Hahn & 2013 & yes & no & 0.04 & yes \\
\hline Mewton & 2013 & yes & no & 0 & yes \\
\hline Sorensson & 2013 & yes & no & 0 & yes \\
\hline Dong & 2014 & yes & unclear & 0 & yes \\
\hline Limalanathan & 2014 & yes & no & 0.08 & yes \\
\hline Waltenberger & 2014 & yes & single-blind & 0.13 & yes \\
\hline
\end{tabular}

and $\mathrm{CK}-\mathrm{MB}$, which are associated with infarct size. Compared with the Con group, the Poc group showed no apparent decrease in the level of peak CK after AMI [standard mean difference $(\mathrm{SMD})=-0.49 ; 95 \%$ confidence interval (CI), -1.09 to-0.1; I2 $=91 \% ; P=0.11$ ). There was substantial between-study heterogeneity (Cochran Q test, $P<0.00001, I^{2}=91 \%$ ). Subgroup analysis based on the method of PCI (PCI was performed only by direct stenting in some studies,and by other methods including direct stenting,balloon dilatation, and thrombus aspiration in rest of studies) showed that not only the decrease in CK became more significant but the heterogeneity also dropped with direct stenting (SMD = -0.82 ; $95 \% \mathrm{CI},-1.18$ to $\left.-0.47 ; I^{2}=64 \% ; P<0.00001\right)$ as compared with other methods $(\mathrm{SMD}=0.96 ; 95 \% \mathrm{CI}$, -0.66 to $2.58 ; I^{2}=96 \% ; P=0.25$ ) (Fig. 2). The CK-MB result was similar to those of CK (Fig. 3). The funnel plots with respect to the end point of CK and CK-MB showed no significant publication bias (Fig. 4). Publication bias measured by Egger's test was not significant $(P=0.21, P$ $=0.68$, respectively). These results showed that Poc can reduce ischemic necrosis of myocardium after acute infarction when patients received direct stenting.

\section{Myocardial infarct size measured by imaging}

The meta-analysis of the studies demonstrated that the perfusion defect index on SPECT or CMR,an estimate of infarct size during $72 \mathrm{~h}$ after AMI, significantly reduced in the Poc group compared to the Con group. The pooled outcome of studies suggested a reduction in IS as measured by imaging ( $\mathrm{SMD}=-0.82 ; 95 \% \mathrm{CI},-1.44$ to $\left.-0.19 ; \quad I^{2}=91 \% ; \quad P=0.01\right)$. There was substantial between-study heterogeneity (Cochran Q test, $P<$ 0.00001, $I^{2}=91 \%$ ) during $72 \mathrm{~h}$ after AMI. Subgroup analysis based on direct stenting showed that the heterogeneity dropped with direct stenting $(\mathrm{SMD}=-0.6 ; 95 \%$ CI, -1.09 to $-0.11 ; I^{2}=75 \% ; P=0.02$ ) as compared with other methods (SMD $=-1.12 ; 95 \% \mathrm{CI},-2.9$ to $0.65 ; I^{2}=$ $96 \% ; P=0.22$ ) (Fig. 5). However, no significant difference was noted between the two groups more than 4 months after AMI (SMD $=-0.43 ; 95 \% \mathrm{CI},-0.9$ to $\left.-0.04 ; I^{2}=87 \% ; P=0.08\right)$, while there was a trend 


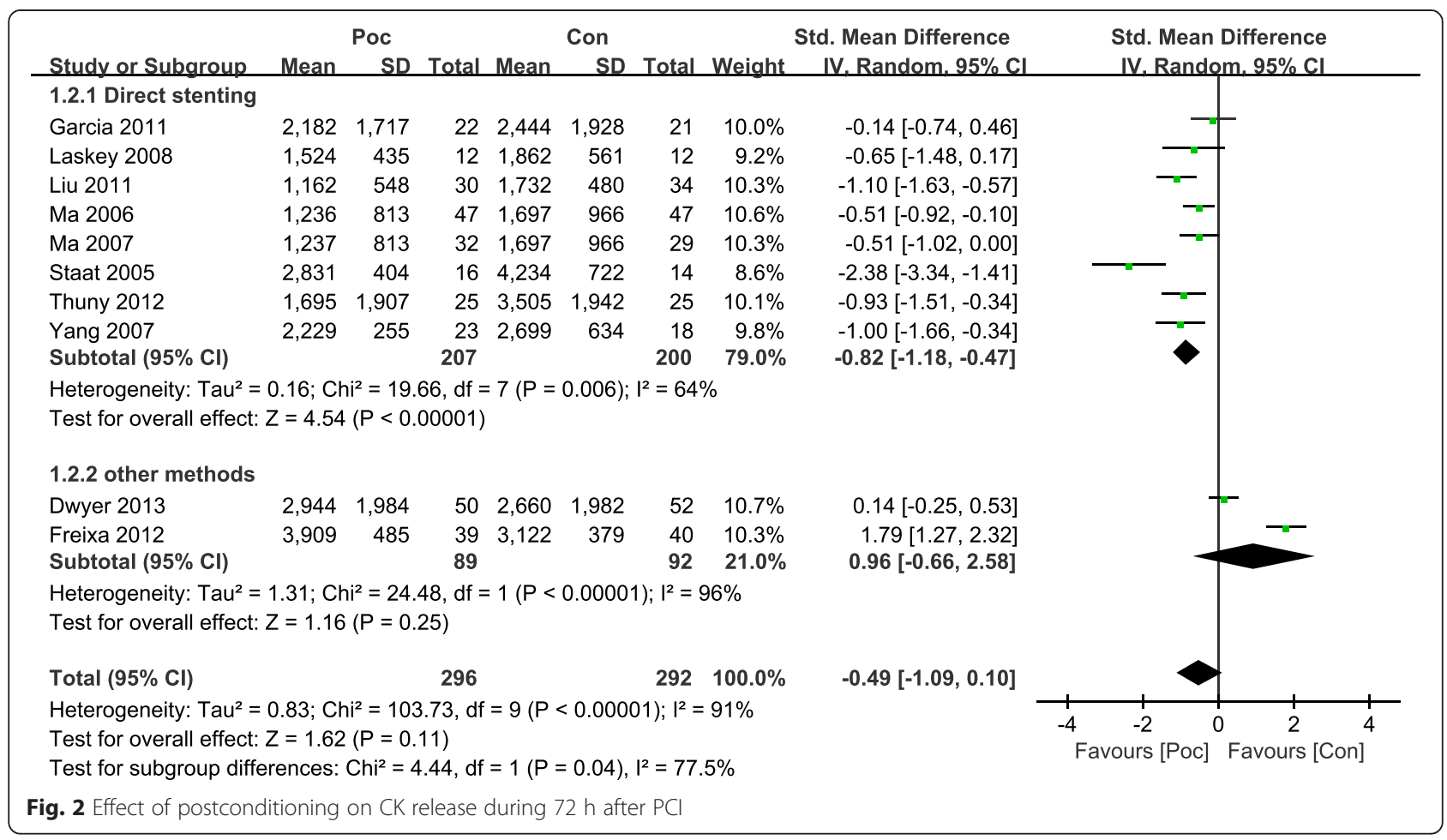

toward the reduction of IS. Subgroup analysis based on direct stenting showed that the heterogeneity dropped with direct stenting $(\mathrm{SMD}=-0.16 ; 95 \% \mathrm{CI}$, -0.43 to $\left.-0.11 ; I^{2}=16 \% ; P=0.24\right)$ as compared with other methods $(\mathrm{SMD}=-0.61 ; 95 \% \mathrm{CI},-0.1 .5$ to 0.28 ; $I^{2}=93 \% ; P=0.18$ ) (Fig. 6). No significant change in heterogeneity was noted when the studies were grouped based on geographic region.

\section{Cardiac function measures}

LVEF was significantly improved in the Poc group in the short term within 7 days after $\mathrm{AMI}(\mathrm{SMD}=0.41 ; 95 \% \mathrm{CI}$,

\begin{tabular}{|c|c|c|c|c|c|c|c|c|c|}
\hline \multirow[b]{2}{*}{ Study or Subgroup } & \multicolumn{2}{|r|}{ Poc } & \multicolumn{3}{|r|}{ Con } & \multirow[b]{2}{*}{ Total } & \multirow[b]{2}{*}{ Weight } & Std. Mean Difference & \multirow{2}{*}{$\begin{array}{l}\text { Std. Mean Difference } \\
\text { IV. Random, } 95 \% \mathrm{Cl}\end{array}$} \\
\hline & Mean & SD & Total & Mean & SD & & & IV. Random, $95 \% \mathrm{CI}$ & \\
\hline \multicolumn{10}{|c|}{ 2.2.1 Direct stenting } \\
\hline Elzbieciak 2013 & 241.6 & 166.8 & 18 & 188 & 240.2 & 0 & & Not estimable & \\
\hline Garcia 2011 & 195 & 33 & 22 & 242 & 40 & 21 & $12.6 \%$ & $-1.26[-1.92,-0.60]$ & \\
\hline Liu 2011 & 165 & 70 & 30 & 280 & 99 & 34 & $13.6 \%$ & $-1.31[-1.86,-0.77]$ & \\
\hline Ma 2006 & 117 & 76 & 47 & 172 & 93 & 47 & $14.6 \%$ & $-0.64[-1.06,-0.23]$ & \\
\hline Ma 2007 & 117 & 76 & 32 & 172 & 93 & 29 & $13.8 \%$ & $-0.64[-1.16,-0.13]$ & \\
\hline Subtotal $(95 \% \mathrm{Cl})$ & & & 149 & & & 131 & $54.6 \%$ & $-0.92[-1.29,-0.56]$ & \\
\hline \multirow{2}{*}{\multicolumn{10}{|c|}{$\begin{array}{l}\text { Heterogeneity: } \mathrm{Tau}^{2}=0.07 ; \mathrm{Chi}^{2}=5.76, \mathrm{df}=3(\mathrm{P}=0.12) ; \mathrm{I}^{2}=48 \% \\
\text { Test for overall effect: } \mathrm{Z}=4.98(P<0.00001)\end{array}$}} \\
\hline & & & & & & & & & \\
\hline \multicolumn{10}{|l|}{ 2.2.2 other methods } \\
\hline Freixa 2012 & 251 & 28.9 & 39 & 195 & 17.6 & 40 & $13.3 \%$ & $2.32[1.75,2.90]$ & \\
\hline Hahn 2013 & 232 & 172 & 350 & 229 & 204 & 350 & $16.0 \%$ & $0.02[-0.13,0.16]$ & $\Gamma$ \\
\hline Xue 2010 & 232 & 172 & 350 & 229 & 204 & 350 & $16.0 \%$ & $0.02[-0.13,0.16]$ & \\
\hline Subtotal $(95 \% \mathrm{CI})$ & & & 739 & & & 740 & $45.4 \%$ & $0.67[0.02,1.32]$ & \\
\hline \multirow{2}{*}{\multicolumn{10}{|c|}{$\begin{array}{l}\text { Heterogeneity: } \mathrm{Tau}^{2}=0.30 ; \mathrm{Chi}^{2}=59.56, \mathrm{df}=2(\mathrm{P}<0.00001) ; \mathrm{I}^{2}=97 \% \\
\text { Test for overall effect: } Z=2.02(P=0.04)\end{array}$}} \\
\hline & & & & & & & & & \\
\hline Total $(95 \% \mathrm{Cl})$ & & & 888 & & & 871 & $100.0 \%$ & $-0.21[-0.70,0.29]$ & \\
\hline \multicolumn{9}{|c|}{ Heterogeneity: $\mathrm{Tau}^{2}=0.39 ; \mathrm{Chi}^{2}=113.05, \mathrm{df}=6(\mathrm{P}<0.00001) ; \mathrm{I}^{2}=95 \%$} & $\begin{array}{ll}-2 & -1\end{array}$ \\
\hline \multicolumn{9}{|c|}{ Test for overall effect: $Z=0.81(P=0.42)$} & Favours [Poc] Favours [Con] \\
\hline \multicolumn{10}{|c|}{ Test for subgroup differences: $\mathrm{Chi}^{2}=17.62, \mathrm{df}=1(\mathrm{P}<0.0001), \mathrm{I}^{2}=94.3 \%$} \\
\hline g. 3 Effect of postcon & tioning & on $\mathrm{CK}-\mathrm{N}$ & $\mathrm{AB}$ relea & ase durir & $\operatorname{ng} 72$ & after & & & \\
\hline
\end{tabular}




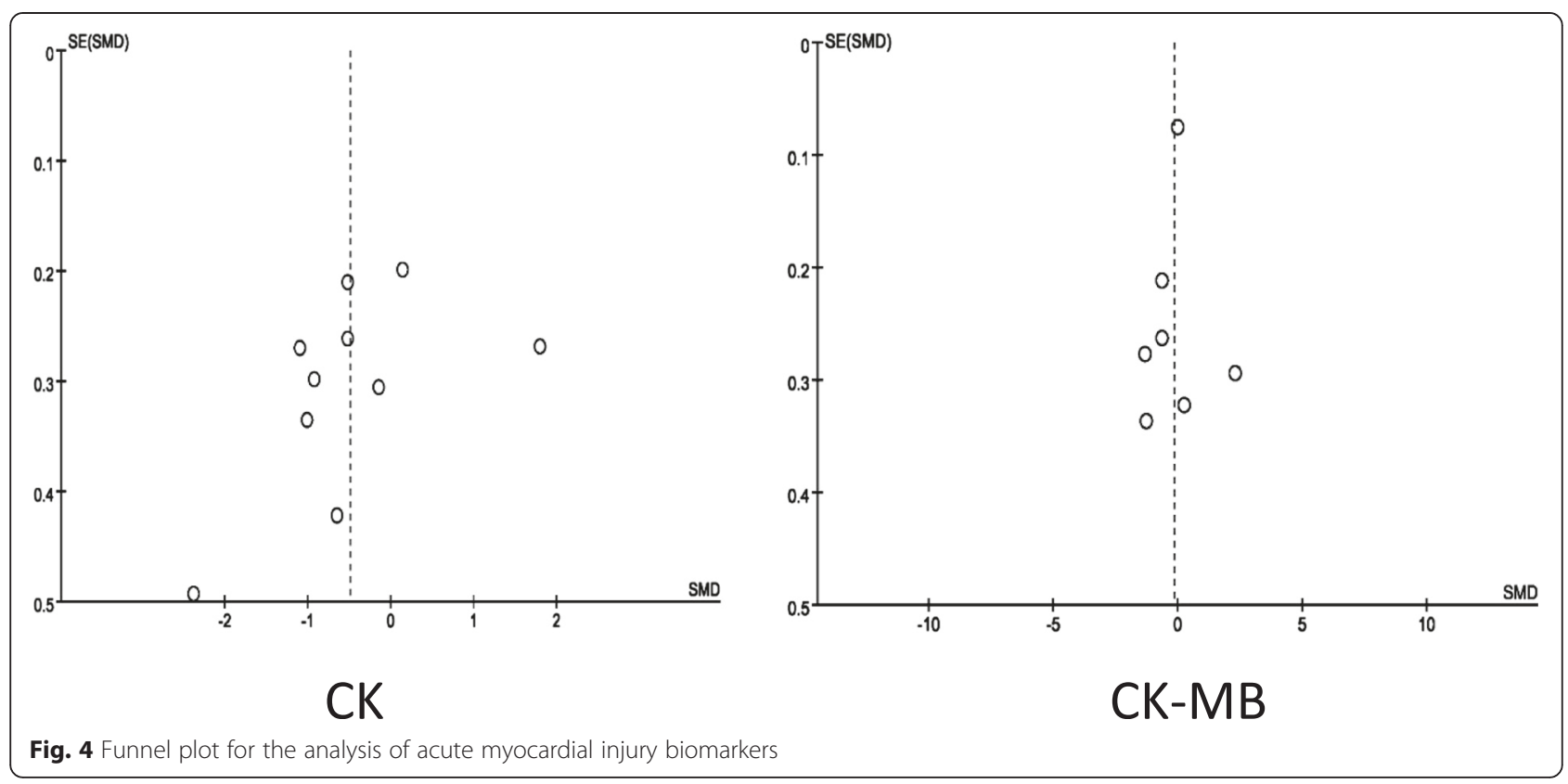

0.16 to $0.65 I^{2}=73 \% ; P=0.001$ ) (Fig. 7). LVEF also improved in the Poc group than in the Con group over 4 months after AMI (SMD $=0.48 ; 95 \% \mathrm{CI}, 0.11$ to $0.85 ; P$ $=0.01$ ). However, subgroup analysis showed that improvement in LVEF was not significant. The heterogeneity dropped with the involvement of direct stenting (SMD $=0.2 ; 95 \% \mathrm{CI},-0.02$ to $0.42 ; I^{2}=0 \% ; P=0.07$ ) as compared with other methods (SMD $=0.57 ; 95 \% \mathrm{CI}, 0.02$ to $1.13 ; I^{2}$ $=88 \% ; P=0.04)$ (Fig. 8). Patients receiving Poc had a lower WMSI (a value of 1 is normal segmental motion and higher values indicate poorer contraction) than those receiving usual primary $\mathrm{PCI}$ in both the short term within 7 days $(\mathrm{SMD}=-2.65 ; 95 \% \mathrm{CI},-4.63$ to $-0.67 ; P=0.009$ ) (Fig. 9) and the long term over 6 months $(\mathrm{SMD}=-3.48$; $95 \%$ CI,-6.47 to $-0.5 ; P=0.02$ ) (Fig. 10).

\section{Discussion}

The current meta-analysis included data from 25 randomized trials involving 2289 participants. The results gave rise to a view that postconditioning following PCI

\begin{tabular}{|c|c|c|c|c|c|c|c|c|c|c|c|}
\hline \multirow[b]{2}{*}{ Study or Subgroup } & \multicolumn{2}{|r|}{ Poc } & \multicolumn{3}{|r|}{ Con } & & & Std. Mean Difference & \multicolumn{3}{|c|}{ Std. Mean Difference } \\
\hline & Mean & SD & Total & Mean & SD & Total & Weight & IV, Random, $95 \% \mathrm{Cl}$ & IV. Rando & $\mathrm{m}, 95 \% \mathrm{Cl}$ & \\
\hline \multicolumn{12}{|l|}{ 3.2.1 direct stenting } \\
\hline Limalanathan 2014 & 16.2 & 12.52 & 120 & 17.4 & 13.04 & 129 & $15.8 \%$ & $-0.09[-0.34,0.16]$ & & & \\
\hline Mewton 2013 & 18 & 9.2 & 25 & 27.6 & 16 & 25 & $14.3 \%$ & $-0.72[-1.30,-0.15]$ & & & \\
\hline Thuny 2012 & 13 & 7 & 25 & 21 & 14 & 25 & $14.3 \%$ & $-0.71[-1.28,-0.14]$ & & & \\
\hline Yang 2007 & 22.8 & 6.7 & 23 & 31.3 & 8.6 & 18 & $13.7 \%$ & $-1.10[-1.76,-0.43]$ & & & \\
\hline Subtotal $(95 \% \mathrm{Cl})$ & & & 193 & & & 197 & $58.1 \%$ & $-0.60[-1.09,-0.11]$ & & & \\
\hline \multicolumn{12}{|c|}{ Heterogeneity: $\mathrm{Tau}^{2}=0.18 ; \mathrm{Chi}^{2}=12.10, \mathrm{df}=3(P=0.007) ; \mathrm{I}^{2}=75 \%$} \\
\hline \multicolumn{12}{|l|}{ 3.2.2 other methods } \\
\hline Freixa 2012 & 27.5 & 17.2 & 39 & 22.1 & 10.2 & 40 & $15.0 \%$ & $0.38[-0.07,0.82]$ & & - & \\
\hline Waltenberger 2014 & 15.4 & 2.1 & 25 & 21.7 & 2.3 & 27 & $13.0 \%$ & $-2.81[-3.59,-2.03]$ & & & \\
\hline Xue 2010 & 13 & 11.2 & 23 & 24.2 & 10.6 & 20 & $13.9 \%$ & $-1.01[-1.65,-0.37]$ & & & \\
\hline Subtotal $(95 \% \mathrm{Cl})$ & & & 87 & & & 87 & $41.9 \%$ & $-1.12[-2.90,0.65]$ & & & \\
\hline \multicolumn{11}{|c|}{ Heterogeneity: $\mathrm{Tau}^{2}=2.36 ; \mathrm{Chi}^{2}=50.81, \mathrm{df}=2(\mathrm{P}<0.00001) ; \mathrm{I}^{2}=96 \%$} & \\
\hline Total $(95 \% \mathrm{Cl})$ & & & 280 & & & 284 & $100.0 \%$ & $-0.82[-1.44,-0.19]$ & & & \\
\hline $\begin{array}{l}\text { Heterogeneity: } \mathrm{Tau}^{2}= \\
\text { Test for overall effect: } \\
\text { Test for subgroup diffe }\end{array}$ & $\begin{array}{l}0.63 ; \mathrm{Ch} \\
Z=2.56 \\
\text { rences: }\end{array}$ & $\begin{array}{l}\mathrm{i}^{2}=64.1 \\
(\mathrm{P}=0.0 \\
\mathrm{Chi}^{2}=0 .\end{array}$ & $\begin{array}{l}\text { 18, df = } \\
01) \\
.31, d f\end{array}$ & $\begin{array}{l}=6(P< \\
=1(P=\end{array}$ & $\begin{array}{r}0.00001 \\
=0.58),\end{array}$ & $\begin{array}{l}1) ; I^{2}=c \\
\left.\right|^{2}=0 \%\end{array}$ & $91 \%$ & & $\begin{array}{ccc}+ & 1 \\
-4 & -2 & 0 \\
& \text { Favours [Poc] }\end{array}$ & \begin{tabular}{|cc}
1 & 2 \\
Favours [Con]
\end{tabular} & 4 \\
\hline Fig. 5 Effect of postcono & tioning & on IS res & eduction & during & g 7 days & after $\mathrm{Pc}$ & & & & & \\
\hline
\end{tabular}




\begin{tabular}{|c|c|c|c|c|c|c|c|c|c|}
\hline \multirow[b]{2}{*}{ Study or Subgroup } & \multicolumn{2}{|r|}{ Poc } & \multicolumn{3}{|r|}{ Con } & & & Std. Mean Difference & \multirow{2}{*}{$\begin{array}{l}\text { Std. Mean Difference } \\
\text { IV, Random, } 95 \% \mathrm{CI}\end{array}$} \\
\hline & Mean & SD & Total & Mean & SD & Total & Weight & IV. Random, $95 \% \mathrm{Cl}$ & \\
\hline \multicolumn{10}{|l|}{ 4.2.1 direct stenting } \\
\hline Elzbieciak 2013 & 25.26 & 12.88 & 18 & 26.18 & 23 & 21 & $13.2 \%$ & $-0.05[-0.68,0.58]$ & \\
\hline Limalanathan 2014 & 13.5 & 8.3 & 120 & 14.4 & 12.52 & 129 & $16.4 \%$ & $-0.08[-0.33,0.16]$ & \\
\hline Thibault 2008 & 11.8 & 10.3 & 17 & 19.5 & 13.3 & 21 & $12.9 \%$ & $-0.63[-1.28,0.03]$ & \\
\hline Subtotal $(95 \% \mathrm{Cl})$ & & & 155 & & & 171 & $42.5 \%$ & $-0.16[-0.43,0.11]$ & \\
\hline \multicolumn{10}{|c|}{$\begin{array}{l}\text { Heterogeneity: } \mathrm{Tau}^{2}=0.01 ; \mathrm{Chi}^{2}=2.38, \mathrm{df}=2(P=0.30) ;\left.\right|^{2}=16 \% \\
\text { Test for overall effect: } Z=1.16(P=0.24)\end{array}$} \\
\hline \multicolumn{10}{|l|}{ 4.2.2 other methods } \\
\hline Dwyer 2013 & 9 & 3 & 50 & 9 & 5 & 52 & $15.4 \%$ & $0.00[-0.39,0.39]$ & \\
\hline Freixa 2012 & 21.8 & 13.2 & 39 & 18.7 & 10.6 & 40 & $14.9 \%$ & $0.26[-0.19,0.70]$ & \\
\hline Lonborg 2010 & 14.7 & 7 & 43 & 17.7 & 7 & 43 & $15.1 \%$ & $-0.42[-0.85,0.00]$ & \\
\hline Waltenberger 2014 & 13.9 & 1.7 & 25 & 18.6 & 2 & 27 & $12.1 \%$ & $-2.49[-3.22,-1.75]$ & \\
\hline Subtotal $(95 \% \mathrm{Cl})$ & & & 157 & & & 162 & $57.5 \%$ & $-0.61[-1.50,0.28]$ & \\
\hline \multicolumn{10}{|c|}{$\begin{array}{l}\text { Heterogeneity: } \mathrm{Tau}^{2}=0.76 ; \mathrm{Chi}^{2}=42.50, \mathrm{df}=3(P<0.00001) ; I^{2}=93 \% \\
\text { Test for overall effect: } Z=1.35(P=0.18)\end{array}$} \\
\hline Total $(95 \% \mathrm{Cl})$ & & & 312 & & & 333 & $100.0 \%$ & $-0.43[-0.90,0.04]$ & \\
\hline \multicolumn{9}{|c|}{$\begin{array}{l}\text { Heterogeneity: } \mathrm{Tau}^{2}=0.34 ; \mathrm{Chi}^{2}=45.79, \mathrm{df}=6(P<0.00001) ; \mathrm{I}^{2}=87 \% \\
\text { Test for overall effect: } Z=1.77(P=0.08) \\
\text { Test for subgroup differences: } \mathrm{Chi}^{2}=0.91, \mathrm{df}=1(P=0.34), \mathrm{I}^{2}=0 \%\end{array}$} & $\begin{array}{ccccc}-2 & -1 & 0 & 1 & 2 \\
\text { Favours [Poc] } & \text { Favours [Con }\end{array}$ \\
\hline \multicolumn{10}{|c|}{ Fig. 6 Effect of postconditioning on IS 4-12months after myocardial infarction } \\
\hline
\end{tabular}

\begin{tabular}{|c|c|c|c|c|c|c|c|c|c|c|}
\hline \multirow[b]{2}{*}{ Study or Subgroup } & \multirow[b]{2}{*}{ Mean } & Poc & \multicolumn{3}{|r|}{ Con } & \multirow[b]{2}{*}{ Total } & \multirow[b]{2}{*}{ Weight } & \multirow{2}{*}{$\begin{array}{l}\text { Std. Mean Difference } \\
\quad \text { IV, Random, } 95 \% \mathrm{Cl}\end{array}$} & \multirow{2}{*}{\multicolumn{2}{|c|}{$\begin{array}{l}\text { Std. Mean Difference } \\
\text { IV. Random, } 95 \% \mathrm{Cl}\end{array}$}} \\
\hline & & SD & Total & Mean & SD & & & & & \\
\hline \multicolumn{11}{|c|}{ 6.2.1 Direct stenting } \\
\hline Dong 2014 & 55.1 & 9.8 & 32 & 42.9 & 10.7 & 30 & $6.8 \%$ & $1.18[0.63,1.72]$ & & \\
\hline Garcia 2011 & 52 & 9 & 22 & 43 & 15 & 21 & $6.2 \%$ & $0.72[0.10,1.34]$ & & \\
\hline Limalanathan 2014 & 51 & 10.37 & 120 & 50 & 13.23 & 129 & $9.3 \%$ & $0.08[-0.17,0.33]$ & & \\
\hline Lin1 2010 & 58 & 3 & 24 & 58 & 3 & 26 & $6.7 \%$ & $0.00[-0.55,0.55]$ & & \\
\hline Liu 2011 & 55 & 8 & 30 & 47 & 10 & 34 & $7.0 \%$ & $0.87[0.35,1.38]$ & & \\
\hline Mewton 2013 & 52 & 8 & 25 & 50 & 12 & 25 & $6.7 \%$ & $0.19[-0.36,0.75]$ & & \\
\hline Tarantini 2012 & 49.4 & 7.4 & 37 & 49.9 & 7 & 38 & $7.6 \%$ & $-0.07[-0.52,0.38]$ & & \\
\hline Thuny 2012 & 52 & 8 & 25 & 50 & 25 & 38 & $7.1 \%$ & $0.10[-0.41,0.60]$ & & \\
\hline Subtotal $(95 \% \mathrm{Cl})$ & & & 315 & & & 341 & $57.3 \%$ & $0.36[0.05,0.67]$ & & \\
\hline \multicolumn{11}{|c|}{$\begin{array}{l}\text { Heterogeneity: } \mathrm{Tau}^{2}=0.13 ; \mathrm{Chi}^{2}=23.64, \mathrm{df}=7(\mathrm{P}=0.001) ; \mathrm{I}^{2}=70 \% \\
\text { Test for overall effect: } Z=2.29(P=0.02)\end{array}$} \\
\hline \multicolumn{11}{|l|}{ 6.2.2 other methods } \\
\hline Dwyer 2013 & 56 & 10 & 50 & 52 & 10 & 52 & $8.1 \%$ & $0.40[0.00,0.79]$ & & \\
\hline Freixa 2012 & 42.7 & 9.8 & 39 & 43.7 & 8.9 & 40 & $7.7 \%$ & $-0.11[-0.55,0.34]$ & & \\
\hline Sorensson 2010 & 50 & 9.63 & 38 & 50 & 11.11 & 38 & $7.6 \%$ & $0.00[-0.45,0.45]$ & & \\
\hline Waltenberger 2014 & 55.3 & 1.9 & 25 & 52.2 & 2.2 & 27 & $6.2 \%$ & $1.48[0.86,2.10]$ & & \\
\hline Xue 2010 & 57 & 9 & 23 & 47 & 11 & 20 & $6.0 \%$ & $0.98[0.35,1.62]$ & & \\
\hline Zhao 2012 & 55.19 & 10.42 & 30 & 51.87 & 9.3 & 32 & $7.1 \%$ & $0.33[-0.17,0.83]$ & & \\
\hline Subtotal $(95 \% \mathrm{Cl})$ & & & 205 & & & 209 & $42.7 \%$ & $0.48[0.04,0.91]$ & & \\
\hline \multicolumn{11}{|c|}{$\begin{array}{l}\text { Heterogeneity: } \text { Tau }^{2}=0.22 ; \mathrm{Chi}^{2}=23.02, \mathrm{df}=5(\mathrm{P}=0.0003) ; \mathrm{I}^{2}=78 \% \\
\text { Test for overall effect: } Z=2.16(P=0.03)\end{array}$} \\
\hline Total $(95 \% \mathrm{Cl})$ & & & 520 & & & 550 & $100.0 \%$ & $0.41[0.16,0.65]$ & & \\
\hline \multicolumn{8}{|c|}{$\begin{array}{l}\text { Heterogeneity: } \text { Tau }^{2}=0.15 ; \mathrm{Chi}^{2}=47.34, \mathrm{df}=13(P<0.00001) ; \mathrm{I}^{2} \\
\text { Test for overall effect: } \mathrm{Z}=3.27(\mathrm{P}=0.001) \\
\text { Test for subgroup differences: } \mathrm{Chi}^{2}=0.19, \mathrm{df}=1(\mathrm{P}=0.67), \mathrm{I}^{2}=0\end{array}$} & & $\begin{array}{lll}-2 & -1 & 0 \\
\text { Favours [Con] }\end{array}$ & $\begin{array}{ccc}0 & 1 & 2 \\
\text { Favours } & {[\mathrm{Poc}]}\end{array}$ \\
\hline Fig. 7 Effect of postcond & litioning & on LVE & F durin & $\ln 7$ days & s after $P$ & & & & & \\
\hline
\end{tabular}




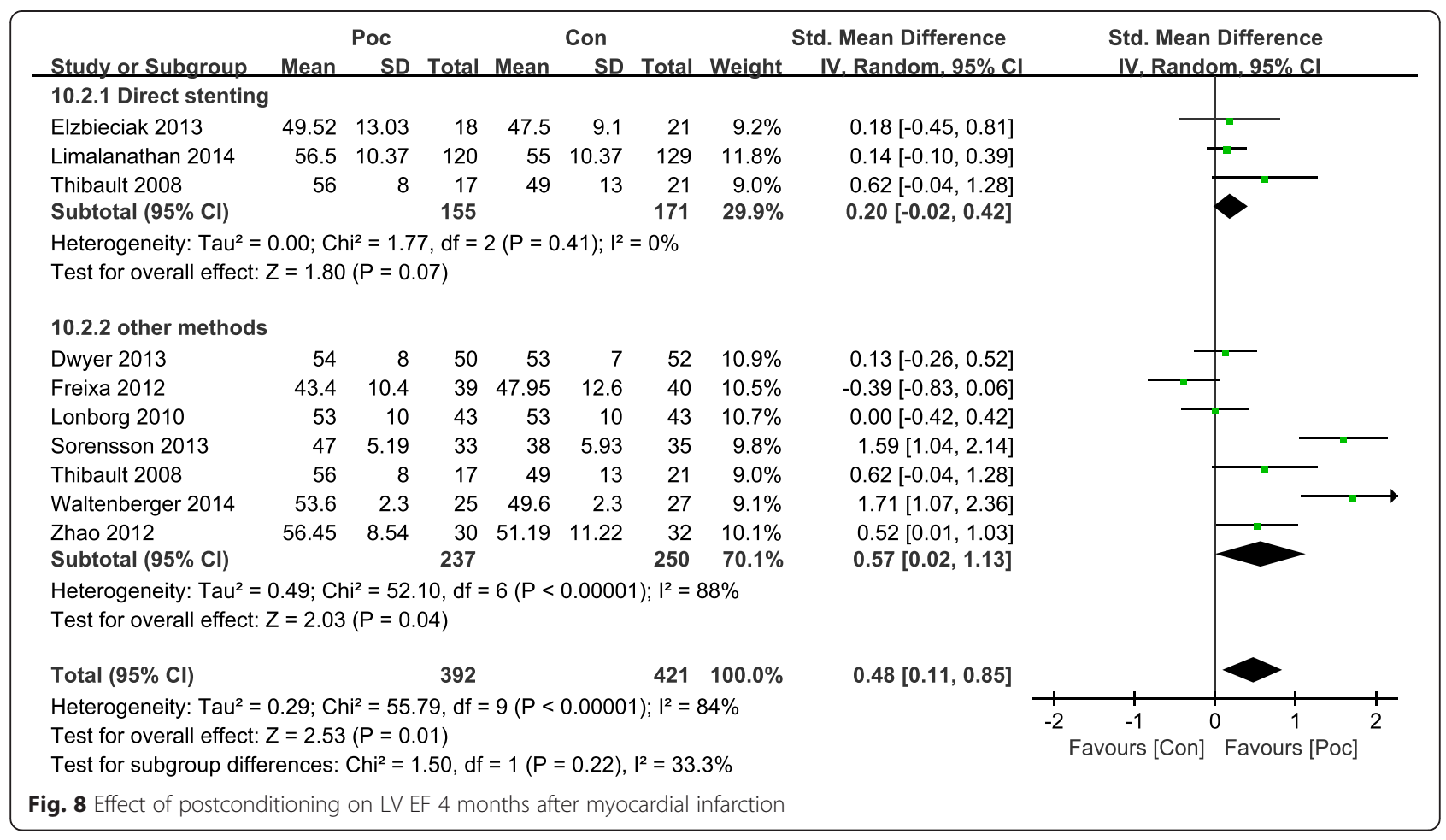

induced by transient coronary ischemia in STEMI patients may reduce myocardial injury biomarkers and improve cardiac function, compared with the usual care group. This cardioprotection was more apparent when direct stenting was performed in PCI compared with other methods of PCI, including balloon angioplasty and thrombus aspiration. The lack of substantial effect of Poc when PCI was completed by angioplasty or thrombus aspiration was most likely due to inadequate revascularization. However, despite the trend in IS reduction, no significant decrease was noted in IS over 4 months after AMI.

Reperfusion therapy is an effective therapeutic approach during the early stage of STEMI patients to prevent heart failure and other cardiovascular events. Many studies have verified that immediate reperfusion is critical to rescue the ischemic myocardium. However, reperfusion has the potential exacerbation of myocardium injury, including myocardial stunning, no reflow, and ventricular arrhythmias [58-60]. Therefore, attenuating reperfusion injury has become an urgent challenge for salvaging myocardium during reperfusion in STEMI patients. Poc performed during angioplasty is technically simple and safe, for cycles of "ischemia/reperfusion" can be easily achieved by repeatedly deflating and inflating the balloon in the culprit artery. A number of studies [7-9, 38-44, 46, 48, 50, 54, 56, 57] described salutary effects of Poc on ischemic/reperfusion injury. In contrast, other studies $[10,11,45,47,49,51-53,55]$ show negative effect of postconditioning, even harmful for myocardium salvage. These studies present high heterogeneity of result due to the difference of Poc protocol, measurement, culprit artery, chest pain elapsed time, endpoints, and the type of PCI. For example, IS can be measured by SPECT or CMR, assessed by percentage of the area at risk, a percentage of the left ventricular mass,or in

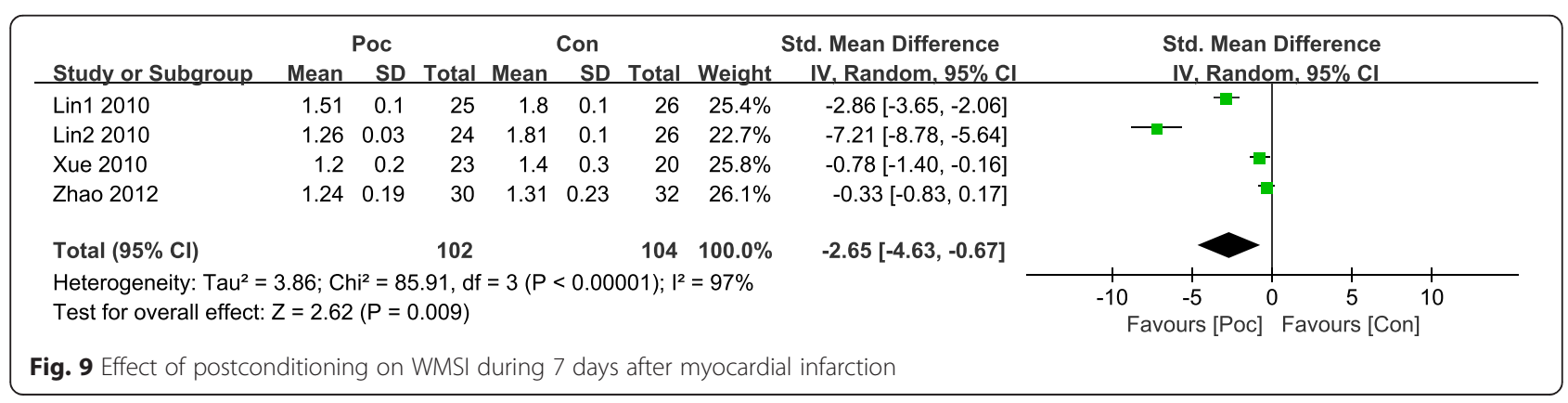




\begin{tabular}{|c|c|c|c|c|c|c|c|c|c|}
\hline \multirow[b]{2}{*}{ Study or Subgroup } & \multicolumn{2}{|r|}{ Poc } & \multicolumn{3}{|r|}{ Con } & & & Std. Mean Difference & \multirow{2}{*}{$\begin{array}{l}\text { Std. Mean Difference } \\
\text { IV. Random. } 95 \% \mathrm{CI}\end{array}$} \\
\hline & Mean & SD & Total & Mean & SD & Total & Weight & IV, Random, $95 \% \mathrm{Cl}$ & \\
\hline Lin1 2010 & 1.27 & 0.05 & 25 & 1.53 & 0.09 & 26 & $33.6 \%$ & $-3.50[-4.39,-2.60]$ & 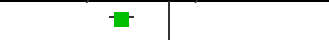 \\
\hline Lin2 2010 & 1.1 & 0.02 & 24 & 1.53 & 0.09 & 26 & $32.1 \%$ & $-6.37[-7.79,-4.96]$ & - \\
\hline Zhao 2012 & 1.2 & 0.17 & 30 & 1.4 & 0.32 & 32 & $34.3 \%$ & $-0.76[-1.28,-0.25]$ & - \\
\hline Total $(95 \% \mathrm{Cl})$ & & & 79 & & & 84 & $100.0 \%$ & $-3.48[-6.47,-0.50]$ & \\
\hline \multicolumn{9}{|c|}{$\begin{array}{l}\text { Heterogeneity: } \mathrm{Tau}^{2}=6.70 ; \mathrm{Chi}^{2}=69.20, \mathrm{df}=2(P<0.00001) ; \mathrm{I}^{2}=97 \% \\
\text { Test for overall effect: } Z=2.29(P=0.02)\end{array}$} & $\begin{array}{lllll}-10 & -5 & 0 & 5 & 10 \\
\text { Favours }[\text { Poc] } & \text { Favours } & \text { [Con] }\end{array}$ \\
\hline
\end{tabular}

grams. A pooled analysis of RCTs has shown that involvement of the left anterior descending (LAD) is one of the strongest predictors of IS [61]. This study revealed that postconditioning presents cardioprotection in patients with STEMI, especially in whom PCI was performed by direct stenting. The reason may be that reperfusion is more adequate by direct stenting than other methods. Appropriate trials are needed to answer this question. Eleven studies performed follow-up from 3 months to 3.4 year [10, 11, 41, 42, 44, 47, 50-52, 55, 57]. The short-term (within 7 days after PCI) beneficial effect in the Poc group included reduced biomarkers of myocardium injury, reduced IS measured by imaging,and the left ventricular function assessed by LVEF or WMSI. Unfortunately, the remarkable decrease of IS did not appear after the long-term follow up. In both short- or long-term follow-up, the global left ventricular function as determined by LVEF improved. Although the analysis suggest that regional left ventricular function assessed by WMSI showed positive result in both short- and long-term follow-up,only several studies cover WMSI. Due to the limited sample size, the results should not be considered conclusive.

Poc could improve myocardial reperfusion in patients with ST-elevation AMI undergoing PCI by reducing no reflow. However, the mechanisms of Poc are not clear. Previos study revealed that high concentrations of inorganic phosphate, reactive oxygen species, and reactive nitrogen species are all present during myocardial ischemia and during reperfusion [62]. Due to the importance of oxidative stress and inflammation in atherosclerotic plaques development and Cardiovascular disease progression, therapeutic of antioxidant seems to be very important [63]. So the cardioprotective effect of Poc partly depend on antioxidant and anti-inflammatory.

Moreover, the question is whether different protocols have different cardioprotective effects. Many different protocols of postconditioning existed, such as $60 \mathrm{~s} \times$ 3 circles, $60 \mathrm{~s} \times 4$ circles, $30 \mathrm{~s} \times 4$ circles, and so on, used in available studies. However, only one study tested the hypothesis that postconditioning of $60 \mathrm{~s} \times 3$ was more protective than postconditioning of $30 \mathrm{~s} \times 3$ [42]. Therefore, additional trials of a large scale are needed to determine the optimal protocol. It is also reported that ischemic postconditioning reduced infarct size in normotensive but not hypertensive rat hearts [64, 65]. Experimental animal data suggest that the presence of diabetes and related conditions, such as obesity and metabolic syndrome, may affect the cardioprotective efficacy of both ischemic and pharmacologic postconditioning. Przyklenk et al. [66] found that ischemic postconditioning cannot play a protective role in reducing infarct size by isolated perfused heart of murine model compared with the normoglycemic heart. In a retrospective analysis, postconditioning the human heart by multiple balloon inflations failed to reduce irreversible injury in patients above the age of 65 years [67]. Yellon and colleagues demonstrated a decline in the effectiveness of RISK pathway signaling with age [68]. They suggested that cardioprotection associated with postconditioning may be affected by age, comorbidities, medications, and the method selection of PCI. Therefore, future strategies will need to focus on the quality of reperfusion. Appropriate trial design is required to provide clearer answers.

\section{Limitations}

Compared with previous studies, the analysis of the present study involves a comprehensive literature search including a large number of relevant studies. Postconditioning was associated with a reduction of infarct size as determined by biochemical quantification and imaging. Cardiac function was assessed by LVEF and WMSI. In this meta-analysis, the long-term (over than 3 month) effect of postconditioning was analyzed as well as the short-term effect. Besides, method selection of PCI was based on subgroup analysis. However, the limitations inherent to the studies contained in the analysis impact the present-study results, such as small sample size, heterogeneity between studies, and risk of performance bias.

\section{Conclusion}

Ischemic postconditioning during PCI in STEMI seems to be superior to the conventional PCI alone in reducing acute myocardial injury, infarction size, and left 
ventricular function including global and regional function, especially in patients who have received direct stenting in PCI. Given the limitations of the current available evidence, additional data involving potential risk factors of restricting postconditioning from large RCTs are needed.

\section{Competing interests}

The authors declare that they have no competing interests.

\section{Authors' contributions}

Yining Yang put forwad the design. Jing Gao and Junyi Luo completed the work of data analysis. Yingying Zheng, Bangdang Chen and Qingjie Chen performed collecting related papers. The manuscript was drafted by Jing Gao and Fen Liu, and revised by Junyi Luo. Finally, Jing Gao performed submitting. All authors read and approved the final manuscript.

\section{Acknowledgements}

This study was supported by the High Technology Research and Development Projects of Xinjiang (No. 201417102)

\section{Author details}

${ }^{1}$ Department of Cardiology, First Affiliated Hospital of Xinjiang Medical University, Urumqi 830054, P.R., China. ${ }^{2}$ Xinjiang Key Laboratory of Cardiovascular Disease Research, Urumqi 830054, P.R., China. ${ }^{3}$ Department of endocrinology, Fifth Affiliated Hospital of Xinjiang Medical University, Urumqi 830011, P.R.China.

\section{Received: 15 October 2015 Accepted: 6 November 2015}

Published online: 16 November 2015

\section{References}

1. Ito H, Okamura A, Iwakura K, Masuyama T, Hori M, Takiuchi S, et al, Myocardial perfusion patterns related to thrombolysis in myocardial infarction perfusion grades after coronary angioplasty in patients with acute anterior wall myocardial infarction. Circulation. 1996;93:1993-9.

2. Granfeldt A, Lefer DJ, Vinten-Johansen J. Protective ischaemia in patients: preconditioning and postconditioning. Cardiovasc Res. 2009;83:234-46.

3. Crisostomo PR, Wairiuko GM, Wang M, Tsai BM, Morrell ED, Meldrum DR. Preconditioning versus postconditioning: mechanisms and therapeutic potentials. J Am Coll Surg. 2006;202:797-812

4. Hausenloy DJ, Yellon DM. Preconditioning and postconditioning: underlying mechanisms and clinical application. Atherosclerosis. 2009;204:334-41.

5. Fan Q, Yang XC, Liu Y, Wang LF, Liu SH, Ge YG, et al. Postconditioning attenuates myocardial injury by reducing nitro-oxidative stress in vivo in rats and in humans. Clin Sci (Lond). 2011;120:251-61.

6. Zhao WS, Xu L, Wang LF, Zhang L, Zhang ZY, Liu Y, et al. A 60-s postconditioning protocol by percutaneous coronary intervention inhibits myocardial apoptosis in patients with acute myocardial infarction. Apoptosis. 2009;14:1204-11.

7. Yang XC, Liu Y, Wang LF, Cui L, Wang T, Ge YG, et al. Reduction in myocardial infarct size by postconditioning in patients after percutaneous coronary intervention. J Invasive Cardiol. 2007;19:424-30.

8. Thuny F, Lairez O, Roubille F, Mewton N, Rioufol G, Sportouch C, et al. Postconditioning reduces infarct size and edema in patients with ST-segment elevation myocardial infarction. J Am Coll Cardiol. 2012;59:2175-81.

9. Ma XJ, Zhang XH, Li CM, Luo M. Effect of postconditioning on coronary blood flow velocity and endothelial function in patients with acute myocardial infarction. Scand Cardiovasc J. 2006:40:327-33.

10. Freixa X, Bellera N, Ortiz-Perez JT, Jimenez M, Pare C, Bosch X, et al. Ischaemic postconditioning revisited: lack of effects on infarct size following primary percutaneous coronary intervention. Eur Heart J. 2012;33:103-12.

11. Limalanathan S, Andersen GO, Klow NE, Abdelnoor M, Hoffmann P, Eritsland J. Effect of ischemic postconditioning on infarct size in patients with STelevation myocardial infarction treated by primary $\mathrm{PCl}$ results of the POSTEMI (POstconditioning in ST-Elevation Myocardial Infarction) randomized trial. J Am Heart Assoc. 2014;3, e000679.

12. Higgins JP, Thompson SG, Deeks JJ, Altman DG. Measuring inconsistency in meta-analyses. BMJ. 2003;327:557-60.
13. Tarantini G, Favaretto E, Napodano M, Perazzolo Marra M, Cacciavillani L, Babuin $L$, et al. Design and methodologies of the POSTconditioning during coronary angioplasty in acute myocardial infarction (POST-AMI) trial. Cardiology. 2010;116:110-6.

14. Limalanathan S, Andersen GO, Hoffmann P, Klow NE, Abdelnoor M, Eritsland J. Rationale and design of the POSTEMI (postconditioning in ST-elevation myocardial infarction) study. Cardiology. 2010;116:103-9.

15. Laskey WK. Catheterization and cardiovascular interventions : official journal of the Society for Cardiac Angiography \& Interventions. Catheter Cardiovasc Interv. 2005;65:361-7.

16. Crimi G, Pica S, Raineri C, Bramucci E, De Ferrari GM, Klersy C, et al. Remote ischemic post-conditioning of the lower limb during primary percutaneous coronary intervention safely reduces enzymatic infarct size in anterior myocardial infarction: a randomized controlled trial. JACC Cardiovasc Interv. 2013;6:1055-63.

17. Carrasco-Chinchilla F, Munoz-Garcia AJ, Dominguez-Franco A, MillanVazquez G, Guerrero-Molina A, Ortiz-Garcia C, et al. Remote ischaemic postconditioning: does it protect against ischaemic damage in percutaneous coronary revascularisation? Randomised placebo-controlled clinical trial. Heart. 2013;99:1431-7.

18. Prunier F, Angoulvant D, Saint Etienne C, Vermes E, Gilard M, Piot C, et al. The RIPOST-MI study, assessing remote ischemic perconditioning alone or in combination with local ischemic postconditioning in ST-segment elevation myocardial infarction. Basic Res Cardiol. 2014;109:400.

19. Smul TM, Lange M, Redel A, Stumpner J, Lotz CA, Roewer N, et al. Desflurane-induced cardioprotection against ischemia-reperfusion injury depends on timing. J Cardiothorac Vasc Anesth. 2009:23:600-6.

20. Pagel PS, Krolikowski JG, Amour J, Warltier DC, Weihrauch D. Morphine reduces the threshold of helium preconditioning against myocardial infarction: the role of opioid receptors in rabbits. J Cardiothorac Vasc Anesth. 2009;23:619-24.

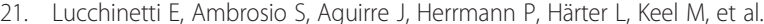
Sevoflurane inhalation at sedative concentrations provides endothelial protection against ischemia-reperfusion injury in humans. Anesthesiology. 2007;106:262-8.

22. Huang Z, Zhong X, Irwin MG, Ji S, Wong GT, Liu Y, et al. Synergy of isoflurane preconditioning and propofol postconditioning reduces myocardial reperfusion injury in patients. Clin Sci (Lond). 2011;121:57-69.

23. Zhang R, Shen L, Xie Y, Gen L, Li X, Ji Q. Effect of morphine-induced postconditioning in corrections of tetralogy of fallot. J Cardiothorac Surg. 2013;8:76.

24. Beck-Schimmer B, Breitenstein S, Bonvini JM, Lesurtel M, Ganter M, Weber A, et al. Protection of pharmacological postconditioning in liver surgery: results of a prospective randomized controlled trial. Ann Surg. 2012;256:837-44. discission 844-835.

25. Loukogeorgakis SP, Panagiotidou AT, Yellon DM, Deanfield JE, MacAllister RJ. Postconditioning protects against endothelial ischemia-reperfusion injury in the human forearm. Circulation. 2006;113:1015-9.

26. Deftereos S, Giannopoulos G, Tzalamouras V, Raisakis K, Kossyvakis C, Kaoukis A, et al. Renoprotective effect of remote ischemic post-conditioning by intermittent balloon inflations in patients undergoing percutaneous coronary intervention. J Am Coll Cardiol. 2013;61:1949-55.

27. Luo W, Li B, Chen R, Huang R, Lin G. Effect of ischemic postconditioning in adult valve replacement. Eur J Cardiothorac Surg. 2008;33:203-8.

28. Dragoni S, Di Stolfo G, Sicuro S, Lisi M, Parker JD, Forconi S, et al. Postconditioning fails to prevent radial artery endothelial dysfunction induced by ischemia and reperfusion: evidence from a human in vivo study. Can J Physiol Pharmacol. 2006;84:611-5.

29. Li B, Chen R, Huang R, Luo W. Clinical benefit of cardiac ischemic postconditioning in corrections of tetralogy of Fallot. Interact Cardiovasc Thorac Surg. 2009;8:17-21.

30. Ji Q, Mei Y, Wang X, Feng J, Wusha D, Cai J, et al. Effect of ischemic postconditioning in correction of tetralogy of Fallot. Int Heart J. 2011;52:312-7.

31. Chen R, Luo WJ, Jiang HH, Long L, Huang RM, Luo FY. Cardiopulmonary protection of ischemic postconditioning in cardiac surgery in children with tetralogy of Fallot. Zhongguo Dang Dai Er Ke Za Zhi. 2009;11:273-6.

32. Durdu S, Sirlak M, Cetintas D, Inan MB, Eryilmaz S, Ozcinar E, et al. The efficacies of modified mechanical post conditioning on myocardial protection for patients undergoing coronary artery bypass grafting. J Cardiothorac Surg. 2012;7:73. 
33. Kim JC, Shim JK, Lee S, Yoo YC, Yang SY, Kwak YL. Effect of combined remote ischemic preconditioning and postconditioning on pulmonary function in valvular heart surgery. Chest. 2012;142:467-75.

34. Zhong H, Gao Z, Chen M, Zhao J, Wang F, Li L, et al. Cardioprotective effect of remote ischemic postconditioning on children undergoing cardiac surgery: a randomized controlled trial. Paediatr Anaesth. 2013;23:726-33.

35. Kim WH, Lee JH, Kim GS, Sim HY, Kim SJ. The effect of remote ischemic postconditioning on graft function in patients undergoing living donor kidney transplantation. Transplantation. 2014;98:529-36.

36. Luo W, Zhu M, Huang R, Zhang Y. A comparison of cardiac postconditioning and remote pre-conditioning in paediatric cardiac surgery. Cardiol Young. 2011;21:266-70.

37. Hong DM, Jeon Y, Lee CS, Kim HJ, Lee JM, Bahk JH, et al. Effects of remote ischemic preconditioning with postconditioning in patients undergoing off-pump coronary artery bypass surgery-randomized controlled trial. Circ J. 2012;76:884-90.

38. Staat P, Rioufol G, Piot C, Cottin Y, Cung TT, L'Huillier I, et al. Postconditioning the human heart. Circulation. 2005;112:2143-8.

39. Ma XJ, Zhang XH, Luo M, Li CM, Shao JH. Effects of preconditioning and postconditioning on emergency percutaneous coronary intervention in patients with acute myocardial infarction. Zhonghua Yi Xue Za Zhi. 2007:87:114-7.

40. Laskey WK, Yoon S, Calzada N, Ricciardi MJ. Concordant improvements in coronary flow reserve and ST-segment resolution during percutaneous coronary intervention for acute myocardial infarction: a benefit of postconditioning. Catheter Cardiovasc Interv. 2008;72:212-20.

41. Thibault H, Piot C, Staat P, Bontemps L, Sportouch C, Rioufol G, et al. Longterm benefit of postconditioning. Circulation. 2008;117:1037-44.

42. Lin XM, Zhang ZY, Wang LF, Zhang L, Liu Y, Liu XL, et al. Attenuation of tumor necrosis factor-alpha elevation and improved heart function by postconditioning for 60 seconds in patients with acute myocardial infarction. Chin Med J (Engl). 2010;123:1833-9.

43. Lonborg J, Holmvang L, Kelbaek H, Vejlstrup N, Jorgensen E, Helqvist S, et al. ST-Segment resolution and clinical outcome with ischemic postconditioning and comparison to magnetic resonance. Am Heart J. 2010;160:1085-91.

44. Lonborg J, Kelbaek H, Vejlstrup N, Jorgensen E, Helqvist S, Saunamaki K, et al. Cardioprotective effects of ischemic postconditioning in patients treated with primary percutaneous coronary intervention, evaluated by magnetic resonance. Circ Cardiovasc Interv. 2010;3:34-41.

45. Sorensson P, Saleh N, Bouvier F, Bohm F, Settergren M, Caidahl K, et al Effect of postconditioning on infarct size in patients with ST elevation myocardial infarction. Heart. 2010;96:1710-5.

46. Xue F, Yang $X$, Zhang B, Zhao C, Song J, Jiang T, et al. Postconditioning the human heart in percutaneous coronary intervention. Clin Cardiol. 2010;33:439-44.

47. Garcia S, Henry TD, Wang YL, Chavez IJ, Pedersen WR, Lesser JR, et al. Longterm follow-up of patients undergoing postconditioning during STelevation myocardial infarction. J Cardiovasc Transl Res. 2011;4:92-8.

48. Liu TK, Mishra AK, Ding FX. Protective effect of ischemia postconditioning on reperfusion injury in patients with ST-segment elevation acute myocardial infarction. Zhonghua Xin Xue Guan Bing Za Zhi. 2011;39:35-9.

49. Tarantini G, Favaretto E, Marra MP, Frigo AC, Napodano M, Cacciavillani L, et al. Postconditioning during coronary angioplasty in acute myocardial infarction: the POST-AMI trial. Int J Cardiol. 2012;162:33-8.

50. Zhao CM, Yang XJ, Yang JH, Cheng XJ, Zhao X, Zhou BY, et al. Effect of ischaemic postconditioning on recovery of left ventricular contractile function after acute myocardial infarction. J Int Med Res. 2012;40:1082-8.

51. Dwyer NB, Mikami Y, Hilland D, Aljizeeri A, Friedrich MG, Traboulsi M, et al. No cardioprotective benefit of ischemic postconditioning in patients with ST-segment elevation myocardial infarction. J Interv Cardiol. 2013;26:482-90.

52. Elzbieciak M, Wita K, Grabka M, Chmurawa J, Doruchowska A, Turski M, et al. Effect of postconditioning on infarction size, adverse left ventricular remodeling, and improvement in left ventricular systolic function in patients with first anterior STsegment elevation myocardial infarction. Pol Arch Med Wewn. 2013;123:268-76.

53. Hahn JY, Song YB, Kim EK, Yu CW, Bae JW, Chung WY, et al. Ischemic postconditioning during primary percutaneous coronary intervention: the effects of postconditioning on myocardial reperfusion in patients with STsegment elevation myocardial infarction (POST) randomized trial. Circulation. 2013;128:1889-96.
54. Mewton N, Thibault H, Roubille F, Lairez O, Rioufol G, Sportouch C, et al. Postconditioning attenuates no-reflow in STEMI patients. Basic Res Cardiol. 2013;108:383.

55. Sorensson P, Ryden L, Saleh N, Tornvall P, Arheden H, Pernow J. Long-term impact of postconditioning on infarct size and left ventricular ejection fraction in patients with ST-elevation myocardial infarction. BMC Cardiovasc Disord. 2013;13:22

56. Dong M, Mu N, Guo F, Zhang C, Ren F, Li J, et al. The beneficial effects of postconditioning on no-reflow phenomenon after percutaneous coronary intervention in patients with ST-elevation acute myocardial infarction. J Thromb Thrombolysis. 2014;38:208-14.

57. Waltenberger J, Gelissen M, Bekkers SC, Vainer J, van Ommen V, Eerens F, et al. Clinical pacing post-conditioning during revascularization after AMI. JACC Cardiovasc Imaging. 2014;7:620-6.

58. Kloner RA, Jennings RB. Consequences of brief ischemia: stunning, preconditioning, and their clinical implications: part 2. Circulation. 2001;104:3158-67.

59. Hausenloy DJ, Yellon DM. Preconditioning and postconditioning: new strategies for cardioprotection. Diabetes Obes Metab. 2008;10:451-9.

60. Piper HM, Meuter K, Schafer C. Cellular mechanisms of ischemia-reperfusion injury. Ann Thorac Surg. 2003;75:S644-8.

61. Stone GW, Dixon SR, Grines CL, Cox DA, Webb JG, Brodie BR, et al. Predictors of infarct size after primary coronary angioplasty in acute myocardial infarction from pooled analysis from four contemporary trials. Am J Cardiol. 2007;100:1370-5.

62. Hausenloy DJ, Duchen MR, Yellon DM. Inhibiting mitochondrial permeability transition pore opening at reperfusion protects against ischaemiareperfusion injury. Cardiovasc Res. 2003;60:617-25.

63. Ciccone MM, Cortese F, Gesualdo M, Carbonara S, Zito A, Ricci G, et al. Dietary intake of carotenoids and their antioxidant and anti-inflammatory effects in cardiovascular care. Mediators Inflamm. 2013;2013:782137.

64. Penna C, Tullio F, Moro F, Folino A, Merlino A, Pagliaro P. Effects of a protocol of ischemic postconditioning and/or captopril in hearts of normotensive and hypertensive rats. Basic Res Cardiol. 2010;105:181-92.

65. Wagner C, Ebner B, Tillack D, Strasser RH, Weinbrenner C. Cardioprotection by ischemic postconditioning is abrogated in hypertrophied myocardium of spontaneously hypertensive rats. J Cardiovasc Pharmacol. 2013;61:35-41.

66. Przyklenk K. Efficacy of cardioprotective 'conditioning' strategies in aging and diabetic cohorts: the co-morbidity conundrum. Drugs Aging. 2011;28:331-43.

67. Darling CE, Solari PB, Smith CS, Furman MI, Przyklenk K. 'Postconditioning' the human heart: multiple balloon inflations during primary angioplasty may confer cardioprotection. Basic Res Cardiol. 2007;102:274-8.

68. Whittington HJ, Harding I, Stephenson Cl, Bell R, Hausenloy DJ, Mocanu $\mathrm{MM}$, et al. Cardioprotection in the aging, diabetic heart: the loss of protective Akt signalling. Cardiovasc Res. 2013;99:694-704.

\section{Submit your next manuscript to BioMed Central and take full advantage of:}

- Convenient online submission

- Thorough peer review

- No space constraints or color figure charges

- Immediate publication on acceptance

- Inclusion in PubMed, CAS, Scopus and Google Scholar

- Research which is freely available for redistribution 